\title{
CALCULATION OF THE SIMPLEST CHEMICAL FORMULA FROM THE PERCENTAGE COMPOSITION.
}

\author{
BY O. L. BRAuER, \\ Selma, California.
}

In these times when the student is confronted with such a multiplicity of facts and phenomena, it is a very serious pedagogical mistake to give a process empirically, when its explanation could be understood by the student. Nevertheless, in some dozen or more textbooks I have looked through, I do not recall a single instance where an explanation was given of the method of calculating the simplest chemical formula from the percentages of composition and the atomic weights. The student-mind craves for an explanation of these things. Those students, who do any thinking, always have a feeling of dissatisfaction when their minds have not found a reason for a process. In this particular instance, it seems to me, a simple explanation could easily be given.

Let $\mathrm{P}$ represent the percentage of any element in a compound, $A$, its atomic weight, $\mathrm{N}$, the number of its atoms in the molecule and $\mathrm{M}$, the simplest molecular weight of the compound.

Then $\mathrm{P}=\frac{\mathrm{N} \times \mathrm{A} \times 100}{\mathrm{M}}$ by definition; e. $g$., the percentage of oxygen in $\mathrm{H}_{2} \mathrm{SO}_{4}, \mathrm{P}=\frac{100 \times 4 \times \mathrm{O}}{\mathrm{H}_{2} \mathrm{SO}_{4}}$ or $\mathrm{P}_{\mathrm{h}}=\frac{100 \times 2 \times \mathrm{H}}{\mathrm{H}_{2} \mathrm{SO}_{4}}$. When the per cent sign is dropped, the left side of the equation is really multiplied by 100 so we must multiply the right-hand side by 100 , hence the 100 in the right-hand side. Dividing through by $\mathrm{A}$ gives the equation in a workable form.

$$
\frac{\mathrm{P}}{\mathrm{A}}=\mathrm{N} \times \frac{100}{\mathrm{M}} \text {. }
$$

$\mathrm{P}$, the percentage, is known; $\mathrm{A}$, the atomic weight, is known, so it is possible to calculate $N \times \frac{100}{\mathrm{M}}$ for each element in the compound. Since $\mathrm{M}$ is the same for each product, $\frac{100}{\mathrm{M}}$ is really the highest common divisor of all the values for $N \times \frac{100}{M}$, we get when we use the above equation for each element in the compound.

To calculate the formula from the percentages and atomic weights of the elements in a compound, it is necessary to:

1. Divide each percentage by the atomic weight.

2. Get the highest common divisor, and divide through by 
it. The values left are the number of atoms of each element in the molecule.

Sulphuric acid analyzes for 2.04 per cent hydrogen, 32.65 per cent sulphur, and 65.31 per cent oxygen.

H. $2.04 \div 1=2.04$.

S. $32.65 \div 32=1.02$.

O. $65.31 \div-16=4.08$.

$\mathrm{H}$.

$\mathrm{S}$.

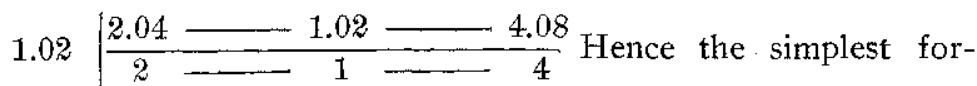
mula is $\mathrm{H}_{2} \mathrm{SO}_{4}$. As a rule, the highest common divisor can be found by inspection. Let us take a harder problem.

Potassium ferrocyanide analyzes for 42.38 per cent $\mathrm{K}, 15.21$ per cent $\mathrm{Fe}, 19.5 \%$ per cent $\mathrm{C}$ and 22.82 per cent $\mathrm{N}$. Dividing each per cent by the atomic weight we get:

$$
\begin{array}{cccc}
\mathrm{K} & \mathrm{Fe} & \mathrm{C} & \mathrm{N} \\
1.08 & .27 & 1.63 & 1.63
\end{array}
$$

Getting the greatest common divisor:

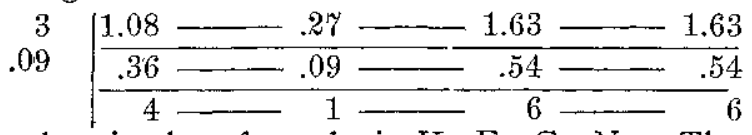

Herice the simplest formula is $\mathrm{K}_{4} \mathrm{Fe}_{\mathrm{e}} \mathrm{C}_{6} \mathrm{~N}_{6}$. The student should be able to calculate the simplest formula for any compound by this method, when the percentages are given.

Editorial Comment on the Above Paper.

The author of the above paper mentions the fact that few if any elementary chemistry texts give any explanation of the derivation of formulas from percentage composition. Perhaps the absence of such work from these texts is commendable rather than otherwise as most texts undertake the consideration of more topics than can be well handled in the time available in most schools. The chemistry editor would like to suggest that a very proper way in which to treat this and similar topics is to have volunteer classes after school for ambitious pupils, in which some of the more difficult subjects are taken up and presented much as the paper in question handles the subject of the calculation of formulas from percentage composition. We would also suggest that for pupils who have not hap physics or who are less mature than seniors, an even simpler mode of treatment than that of the above paper will probably prove more successful. For example, instead of employing a mathematical expression of the 
facts, suppose we simply discuss them in as nearly everyday language as possible. In the case in point, one might say, "If we knew the actual weights in grams of each of the kinds of atoms in this compound and also the actual weights in grams of each of the elements present, we could divide the weight of each element by the weight of its atom and thus find the exact number of atoms present in the given quantity of the compound. After doing this for each element present, we could then relate the numbers of each kind of atom to each other and put them in their lowest terms and thus get the simplest formula for the compound. But we do not accurately know the weight in grams of the individual atoms. Hence we cannot find the actual number present. We do, however, know the relative weights of the atoms. If, now, we should divide the weight of each element present by the relative weight number of its atom, we would not get as our quotient the actual number of atoms present, but if we thus treat each of the elements we will get numbers for quotients which will bear the same numerical relation to each other that the true numbers of atoms bear.

"To show that this method of treatment is correct, let us consider the case of a coffee merchant who buys $\$ 400$ worth of Mocha coffee and $\$ 250$ worth of Java coffee and blends them. Now, if we knew the price per pound paid by him for each kind of coffee, we could speedily tell how many pounds of each kind he put into the blend but in the absence of a knowledge of the price per pound, suppose it is known that the prices paid bear the relation to each other of 8 to 5 , the Mocha coffee being the higher priced. While we cannot yet tell just how many pounds of each the merchant employed, we can find how many of each he used, relative to the other, as follows: If we divide $\$ 400$ (the amount belonging to the Mocha coffee) by 8 (the relative value of Mocha coffee) we get 50 as our quotient. If we next divide $\$ 250$ (the amount belonging to the Java coffee) by 5 (the relative value of Java coffee) we get 50 as a quotient. The weights of Mocha and Java coffee used must then have been to each other as 50 is to 50 which in lowest terms is as 1 is to 1 .

"Having seen while dealing with common things that the method suggested above is correct, let us apply it to find the simplest formula of, say, sulphuric acid. This substance has been analyzed and is found to contain hydrogen, sulphur and oxygen. If 100 grams are taken we find in it 2 grams of hydrogen, 32.7 grams of sulphur and 65.3 grams of oxygen, Now the 
relative weights of the atoms of these three elements are as 1 is to 32 is to 16 approximately. If then, following our practice with the coffee, we divide the amount belonging to each element by the relative number belonging to each element, we will have as quotients numbers which, while not the true numbers of atoms present, will, nevertheless, be in the same numerical relation as the true numbers of the atoms and they may therefore be put in their lowest terms and used to indicate the formula of this substance.

" $2 g / 1$ gives 2 .

"Example.

" $32.7 \mathrm{~g} / 32$ gives 1 (nearly).

"65.3g/16 gives 4 (nearly).

"Hence the numbers of hydrogen, sulphur and oxygen atoms present are to each other as 2 is to 1 is to 4 . As these numbers are already in lowest terms we may say that in sulphuric acid for every sulphur atom there are two hydrogen atoms and four oxygen atoms or $\mathrm{H}_{2} \mathrm{SO}_{4}$."

Other topics that may thus be profitably treated with volunteer classes at the proper timies may be suggested-for example:

1. The atomic theory, what it sought to account for and how it does so.

2. Avogadro's Hypothesis, why he brought it out, how it serves to explain Gay Lussac's Law (also Boyle's and Charles' Laws), the necessary consequences of Avogadro's Hypothesis.

3. Molal volume and its use in finding molecular weights of gaseous substances (an application of Avogadro's Hypothesis).

4. Use of osmotic pressures in finding molecular weights (an application of Avogadro's Hypothesis to the case of dissolved substances).

As other means of finding molecular weights:

5. Elevation of boiling point.

6. Depression of freezing point.

\%. Law of Du Long and Petit and related laws.

The annual statement on the production of abrasive materials in the United States in 1914 is now available for distribution by the Geological Survey. Under this heading are considered only those mineral products which are used for grinding and polishing and other abrasive operations. The value of all the abrasive materials consumed in the United States during the year amounted to $\$ 3,614,263$, 